\title{
Network Interactive Platform Ideological and Political Education Based on Internet Technology
}

\author{
WanRenlong \\ Nanchang Institute of Science \&Technology,Nanchang 330108,China
}

\begin{abstract}
Keywords: network media; ideological and political; interactive platform; Web technology; cloud technology
\end{abstract}

\begin{abstract}
With the continuous development in the information age, the impact of all sorts of new network media on the public is more and more big, especially on young college students. They tend to try and accept new things, so the traditional ideological and political education management mode has been seriously challenged. Under this background, according to the cloud computing platform technology, combined with the Internet and Web technology, Innovative interactive platform for Ideological Education has been put forward, which includes five functional layers and three layers architecture, and can provide powerful online learning, interaction and resource sharing functions for students. The three layer architecture data layer, logic layer and said layer has been designed, taking Visual Studio as the development environment, the ultimate form of the ASPX file. The development of the platform provides a very valuable reference for the innovation of Ideological and political education.
\end{abstract}

\section{Introduction}

Modern information technology is changing people's way of life, the way of study, the way of working and the way of production. In this information era that full of opportunities and challenges, it has become increasingly important how to seize the opportunity to form a new teaching model that combining information technology and school teaching. Therefore, it is an urgent issue need to study and explore that constructing the modern teaching mode based on the network environment, and exploring the interactive learning theory based on the network environment. The interactive teaching mode based on Web can apply the computer network resources and multimedia resources to the teaching in a unified way, and provide audio-visual full range of sensory stimulation, which can make the abstract teaching content more specific. This design is based on the Internet and cloud technology platform, and the development of the ideological education teaching platform, in order to increase the interaction of Ideological and political teaching.

\section{Innovation of Ideological and political education based on cloud technology and Internet}

Since the birth of the Internet and computer, it quickly covered all areas of the world that the rapid development of modern information technology with the core of computers and the Internet in just a few years time, and it also set off a new teaching reform in the field of education, which is mainly reflected in the following two aspects:

(1) The network makes the ideological and political education more exciting Network teaching can make the teaching content more rich, and change the teaching method. Under the network environment, education and teaching work can be unnecessary to have a face to face, 
make teaching melt in a fun environment, which makes education thought some is not easy to face to face teaching task to the development of reasonable, especially for shy students, from a large extent closer to the distance between teachers and students, convenient communication and cooperation between teachers and students.

(2) The network promoted the non - power influence of the ideological and Political Education

Students can spread Ideological and political education by from different levels through QQ, Wechat and WeiBo, which is convenient for teachers to understand students' thoughts, and enable teachers to change the traditional teaching mode, and let oneself become fashionable again, direct real-time communication bridge between teachers and students can be established, so that the teachers set up the non power influence in the ideological and political education.

Cloud computing is the interactive platform for the development of technical support, using cloud technology can achieve mutual learning, communication and sharing, for individuals and organizations to establish a powerful resource sharing interactive learning platform. Teaching platform can greatly improve students' learning enthusiasm, cultivate students' learning interest, thus highlighting the students in the teaching process of the main position, its main function is reflected in the:

(1) the use of advanced cloud computing platform, you can build a communication between teachers and students learning methods, so as to focus on the students in the teaching process of the main status;

(2) according to the powerful resource sharing and storage function of cloud computing, we can establish the ideological and political teaching resources library of teachers and students, enrich the content of the course, and increase the interest of teaching.

(3) the use of cloud computing platform of multimedia function can create powerful graphics, sound and video and Mao's ideological and political education system for students, active classroom atmosphere, which can fully mobilize the enthusiasm of the students learning and class of enthusiasm.

\section{The resource optimization algorithm of the Ideological and Political Education interactive platform}

Interactive platform architecture will produce a large number of database files, in order to make the network resource optimization configuration and need to interactive platform for and generated data, algorithm optimization, the current in the classical algorithm for data classification using more decision algorithm. ID3 algorithm is of a sample of the training data set for learning, resulting in a decision tree and assumptions a data sample the only belongs to accord with the learning goals or not consistent with learning goals, all of the sample data is discrete. For a training example set $E_{s}$, if the ratio of the target to be studied is $P_{+}$, the proportion of non- conforming is $1-P_{+}$, then the entropy formula is formula (1) as follows:

$$
\operatorname{Entropy}\left(E_{s}\right)=-P_{+} \log _{2} P_{+}-P_{-} \log _{2} P_{-}
$$

In formula (1), assuming that $\log _{2} 0=0$, if we use the sample data $A$ for training samples $E_{s}$, the number of packets will be reduced, the new expected value is formula (2) as follows:

$$
\operatorname{NewEntropy}\left(E_{s i}, A\right)=\sum_{i \in \operatorname{Value}(A)}\left(\left|E_{s i}\right| /\left|E_{s}\right|\right) \operatorname{Entropy}\left(E_{s}\right)
$$

In formula (2), $\operatorname{Gain}\left(E_{a i}, A\right)$ is $A$ relative to the $E_{s}$ information, means the amount of Entropy $\left(E_{s}\right)$ reduced, the greater the amount of information, the more favorable for the optimization of the ideological and political education platform resource, the expression is formula (3) as follows: 


$$
\operatorname{Gain}\left(E_{a i}, A\right)=\operatorname{Entropy}\left(E_{s}\right)-\operatorname{NewEntropy}\left(E_{s i}, A\right)
$$

The C4.5 algorithm is improved by the ID3 algorithm. In the C4.5 decision tree, each node of the tree are retained in the calculated value of the attribute information, the information of each value to meet the learning goals and not consistent with the learning objectives. According to the information stored in the decision tree, the attribute of the training set can be judged, and the attribute of the training set is determined by the information entropy of the information in the node. the expression is formula (4) as follows:

$$
\begin{aligned}
& \operatorname{Infox}(S)=-\sum_{i=1}^{k}\left(\operatorname{freq}\left(C_{i}, S\right) /|S|\right) \\
& \log 2\left(\operatorname{freq}\left(C_{i}, S\right) /|S|\right)
\end{aligned}
$$

In formula (4), freq $\left(C_{i}, S\right) /|S|$ is the sample quantity that belong to category $C_{i}$ in the set $S,|S|$ is the sample quantity, the formula for information weighted sum of a sample data is formula (5) as follows:

$$
\operatorname{Infox}(T)=-\sum\left(\left(\left|T_{i}\right| /|T|\right) \operatorname{Info}\left(T_{i}\right)\right)
$$

For the optimization of the database resources of the interactive platform of Ideological and political education, the specific implementation steps of the C4.5 algorithm are shown in Fig.1.

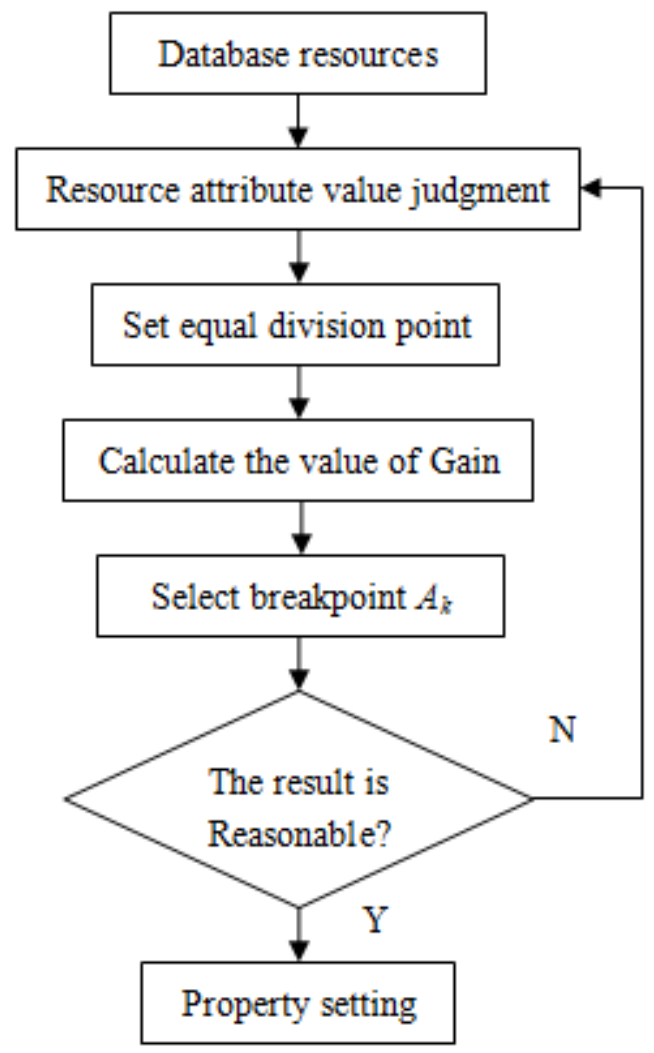

Fig. 1 The flow chart of platform database resource optimization

Fig. 1 is the flow chart of the Ideological and political education interactive platform database resources optimization. The main steps can be summarized to 4 steps as follows:

(1) Find the minimum value of the ideological and political education interactive platform database resources, the value assigned to the min, looking for the maximum value of the data sample properties, the value assigned to max; 
(2) Setting the $N$ equal points in the interval [min, max], $(i=1,2, \ldots, N)$;

(3)Calculation of $\left[\mathrm{min}, A_{i}\right]$ and $\left(A_{i}, \max \right)(i=1,2, \ldots, N)$ interval Gain value, and the calculation of the numerical value for comparison;

(4) Select $A_{k}$ that has the maximum Gain value as the breakpoint, and set the property to interval values of [min, $\mathrm{Ai}]$ and $\left(A_{i}, \max \right)$.

\section{Framework of Ideological and Political Education interaction platform based on Internet cloud platform technology}

It needs vast data resources support and a lot of Internet sharing resource to architecture in Ideological and political education interactive platform based on Internet platform technology, this requires the help of the current cloud computing environment. A cloud computing platform is based on IaaS, the user can rent SaaS software applications that developed by PaaS, so as to maximally meet the individual needs of a large number of users, the basic framework of its structure as shown in figure 2.

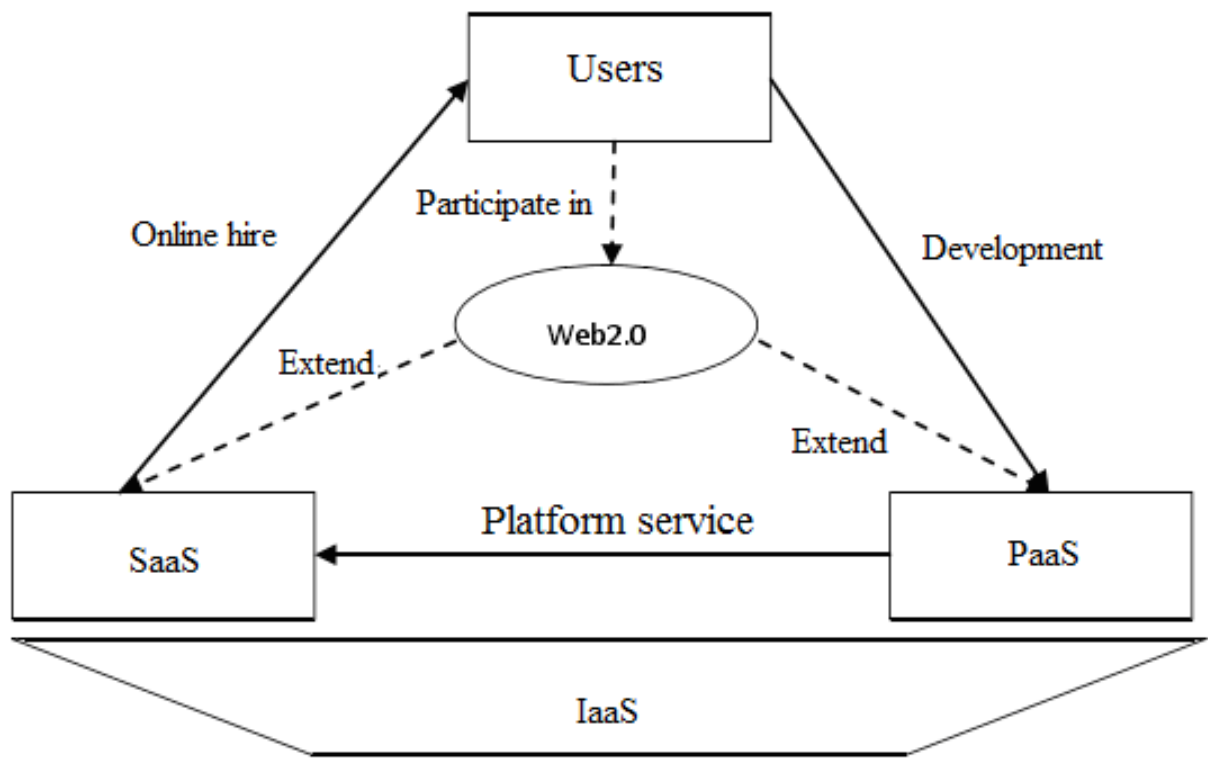

Fig.2 The basic architecture of cloud services platform

Fig.2 shows the basic structure of the ideological and political teaching platform interactive cloud platform, the PaaS generally includes the infrastructure that required for the operation of the application, such as server instance and the ability to store the instance, IaaS, PaaS, SaaS can provide users with different services based on the same architecture. The ideological and political education interactive platform in this research is mainly based on the development of Web teaching system, its main function is divided into five modules, and interactive function layers as shown in Fig.3.

The interactive platform function mainly includes five levels, user management in which is mainly registration and login service for students and teachers account; the resource construction and sharing can authorize the course of PPT and CAI courseware and teaching video in the teaching system uploading and save to the server, and the establishment of curriculum self-study database; network on-line teaching can achieve real-time online teaching function through Web and LAN; Interactive learning community forum function as BBS and the message board function, facilitate interactive learning and communication between teachers and students; students' autonomous 
learning mainly can choose learning resources of Ideological and political education, and autonomous learning and testing for students with different interests.

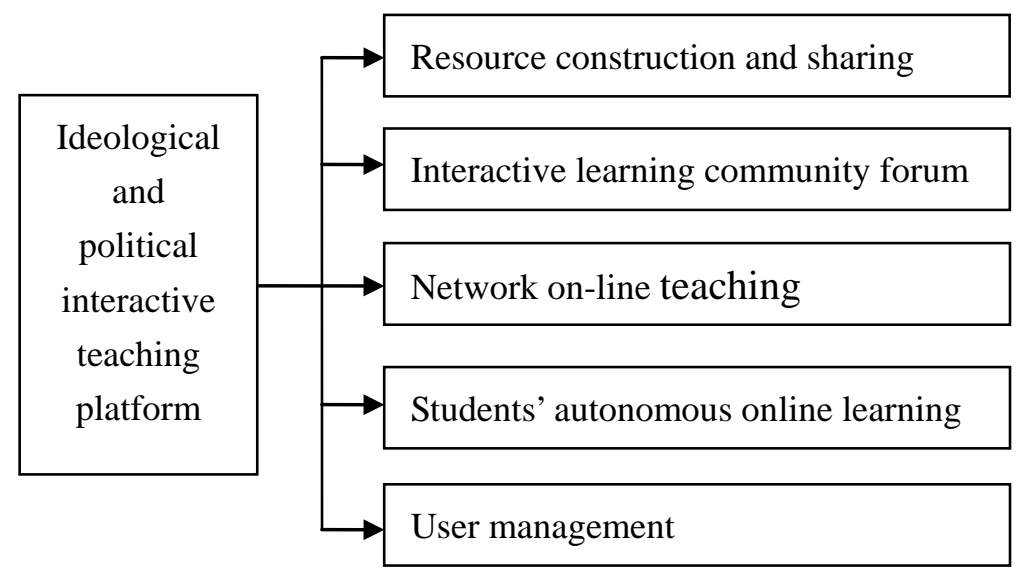

Fig. 3 The function level interactive platform

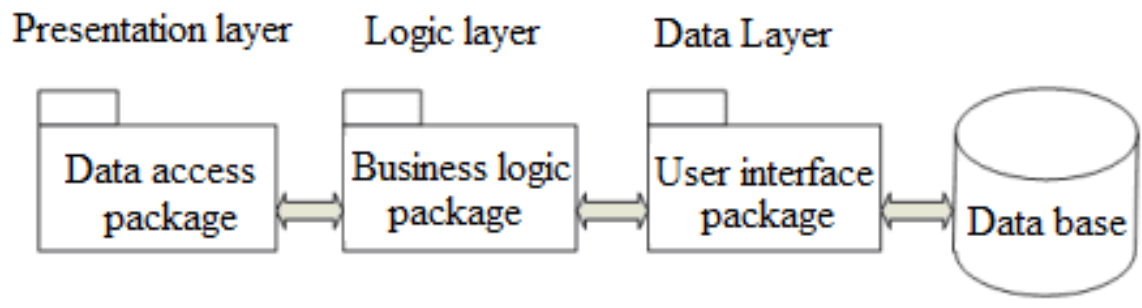

Fig. 4 Three layers structure schematic diagram of the interactive platform

The network ideological and political education interaction platform based on Internet technology is mainly divided into three levels, including data layer, logic layer and presentation layer. Data layer is mainly responsible for data storage, and interactive components service, logic layer can mainly provide platform of Ideological and political education related services according to the different business rules, presentation layer can provide related services after obtaining the information through the logic layer. The development environment is visual studio, which mainly in the form of ASPX file.

\section{Conclusions}

In order to innovate Ideological and political teaching mode and teaching content, the three layers of platform architecture is designed according to the five levels functional requirements, combining with Internet technology and Web technology, and the modern ideological and political education interactive platform has been built based on the cloud platform. The feasibility of the platform is verified with Studio Visual as the development environment, and with the expression form of ASPX documents. In the future teaching, network and multimedia will occupy the dominant position, so in Ideological and political teaching, using cloud computing platform is the future trend, cloud technology will provide strong technical support for the development of the optimal allocation of teaching resources and interactive platform.

\section{Reference}

[1] Zhao Mingjun. Talk about how to build a "Trinity" of the ideological and political education mode [J]. Hunan Tide History of the CPC in Hunan, 2010 (9):108-109 
[2] Ji Haiju. In the ideological and Political Education: micro-blog blocked or hand [J]. Academia Bimestris, 2011(5):196-200.

[3] Zhang Zhongdi. A review of the research on the construction of campus culture and ideological and political education in Colleges and Universities [J]. Forum on Contemporary Education , 2009(1):114-11

[4] Zhang Qin. Design and application prospect of enterprise information system application framework based on cloud computing and [J]. China Offshore Oil and Gas, 2013.25 (01): 91-94.

[5] Zhang Yunxia. Analysis of ERP enterprise management information system based on cloud computing [J]. Information Security and Technology, 2013.36 (02): 47-49.

[6] Zhang Xiaojuan, easy Mingwei. Cloud computing and SOA enterprise integration architecture and implementation [J]. Computer system \& application. 2011,20(09): 1-6.

[7] Yang Nan, Li Dongbo, Tong Yifei. Research on cloud computing ERP architecture for services [J]. Manufacturing Automation, 2012, 34(19): 74-77.

[8] Wu Jia. Small and medium enterprises to implement the implementation of cloud computing ERP [J]. China Packaging Industry, 2012 (09): 49-50.

[9] Wang Lian, Huang Xin, Ma Fei. Study on the design of small and medium sized logistics enterprise cloud service system [J].Logistics Technology, 2013, 32(01): 234-236.

[10] Lv Lin. Research on the development of digital manufacturing technology at home and abroad [J]. industry perspective. 2009, 76 (3): 2-4

[11] Gao Qun, Dong Lei. The design of quality management module of ERP [J]. measurement and testing technology, 2013,40 (03): 55-58.

[12] SaaS ERP: Network and information[J]. Network \& Information, 2012,26 (07): 51.

[13] Zhang Yunxia. Analysis of ERP enterprise management information system based on cloud computing [J]. Information Security and Technology, 2013,36 (02): 47-49.

[14] He Yao, Wang Wenqing, Sheffi. Research on massive data mining based on cloud computing [J]. Computer Technology and Development, 2013, 23 (2): 69-72

[15] Ding Yan, Yang Qingping, Qian Yuming. Research on the architecture and key technologies of data mining platform based on cloud computing [J]. ZTE technology Journal, 2013,19 (1): 53-60

[16] Liang Shuang. Research and implementation of cloud computing framework model based on SOA [J]. Computer Engineering and Application, 2011,47(35): 92-94.

[17] Kang Ruifeng. Research on Paas middleware based on SOA architecture [J]. Computer knowledge and technology, 2013.9 (07): 1537-1539.

[18] Li Qi, Zhu Qinghua. Enterprise informatization strategic planning based on the integration of SOA and cloud computing [J]. Journal of Intelligence, 2011,3 (30): 147-150. 